\title{
Microwave-Assisted Synthesis of Fluorescent Pyrido[2,3-b]indolizines from Alkylpyridinium Salts and Enaminones
}

\author{
Ekaterina A. Sokolova ${ }^{1}$, Alexey A. Festa ${ }^{1}$, Karthikeyan Subramani ${ }^{1}{ }^{1}$, Victor B. Rybakov ${ }^{2}$, \\ Alexey V. Varlamov ${ }^{1}$, Leonid G. Voskressensky ${ }^{1}$ and Erik V. Van der Eycken ${ }^{1,3, *}$ \\ 1 Organic Chemistry Department, Science Faculty, RUDN University, Miklukho-Maklaya st., 6, \\ 117198 Moscow, Russia; soko-katya@mail.ru (E.A.S.); festa_aa@pfur.ru (A.A.F.); \\ subramanikarthikeya91@gmail.com (K.S.); avarlamov@sci.pfu.edu.ru (A.V.V.); \\ lvoskressensky@sci.pfu.edu.ru (L.G.V.) \\ 2 Department of Chemistry, Lomonosov Moscow State University, Leninskie Gory, 1-3, \\ 119991 Moscow, Russia; rybakov20021@yandex.ru \\ 3 Laboratory for Organic \& Microwave-Assisted Chemistry (LOMAC), Department of Chemistry, \\ University of Leuven (KU Leuven), Celestijnenlaan 200F, B-3001 Leuven, Belgium \\ * Correspondence: erik.vandereycken@kuleuven.be
}

Academic Editors: Alessandro Ponti and Giorgio Molteni

Received: 1 August 2020; Accepted: 3 September 2020; Published: 5 September 2020

\begin{abstract}
Pyridinium ylides are well recognized as dipoles for cycloaddition reactions. In its turn, the microwave-assisted interaction of $\mathrm{N}$-(cyanomethyl)-2-alkylpyridinium salts with enaminones unexpectedly proceeds as a domino sequence of cycloisomerization and cyclocondensation reactions, instead of a 1,3-dipolar cycloaddition. The reaction takes place in the presence of sodium acetate as base and employs benign solvents. The optical properties of the resulting pyrido[2,3-b]indolizines were studied, showing green light emission with high fluorescence quantum yields.
\end{abstract}

Keywords: pyridinium ylide; indolizine; domino reaction; fluorescence

\section{Introduction}

Indolizines, and in particular the annulated ones, are frequently found to exhibit useful biological [1-6] and optical properties [7-14]. The synthesis of indolizines usually relies on the reactivity of pyridinium ylides, which can undergo cycloaddition reactions with alkenes [15-18] or alkynes [19-23]. In some cases, interaction of ylides with alkenes or alkynes leads to a different outcome [24-26]. Another approach towards the indolizine scaffold is based on intramolecular cyclization of 2-alkylpyridinium ylides. For instance, Opatz et al. used 2-alkyl-1-(cyanomethyl)pyridinium salts to prepare aminoindolizines [27]. Following our interest in the chemistry of cyanomethylpyridinium salts [28-30], we recently showed that the interaction of $N$-(cyanomethyl)pyridinium chlorides with enaminones under basic conditions proceeds as a pseudo-three-component reaction, resulting in the formation of pyridoindolizine-10-carbonitriles [31].

In this work we discovered that the reactions of 2-alkyl- $N$-(cyanomethyl)pyridinium salts $\mathbf{1}$ with enaminones 2 proceed unexpectedly as a two-component domino sequence of cycloisomerization and cyclocondensation reactions, while cycloaddition processes were not observed (Scheme 1). 


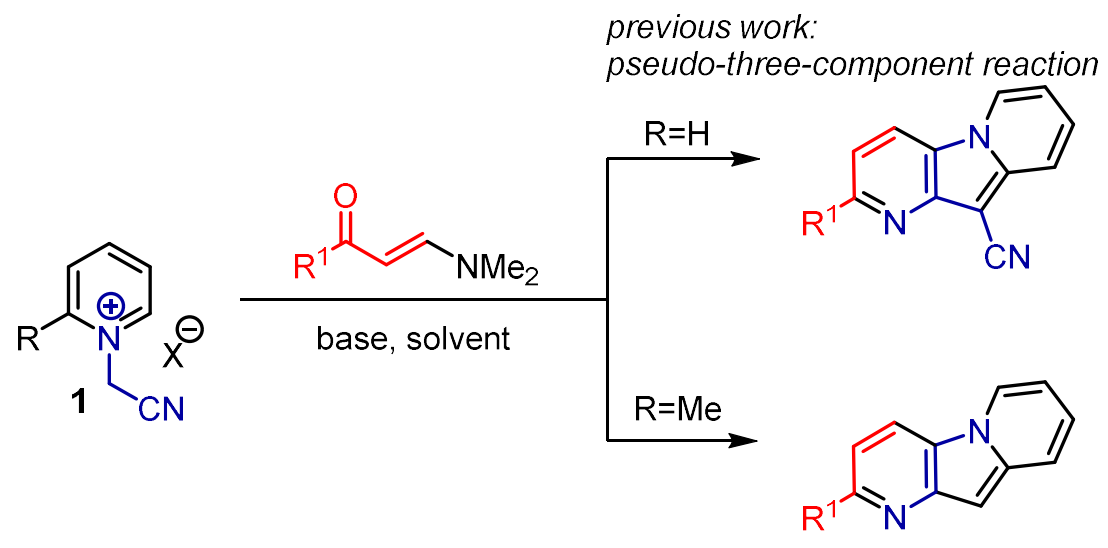

this work: two-component reaction

Scheme 1. General representation of the current work.

\section{Results}

Based on the previously investigated reaction [31], we started optimization of the conditions with the use of sodium acetate as base and an iso-propanol/water mixture as solvent (Table 1, entries 1-6). Varying the ratio of the starting materials and the base, the target compound 3a was obtained with $50 \%$ yield (Table 1, entry 5). The use of other bases such as $\mathrm{Et}_{3} \mathrm{~N}$, DIPEA, or $\mathrm{NH}_{4} \mathrm{OAc}$ did not improve the yield (Table 1, entries 7-9). Inorganic bases did not ameliorate the process either (Table 1, entries 10 and 11). The variation of reaction time or temperature commonly led to diminished yields (Table 1 , entries 12-14).

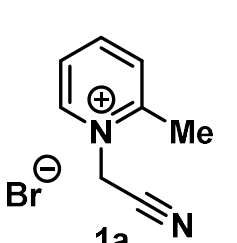

Table 1. Reaction conditions optimization.

$1 \mathrm{a}$

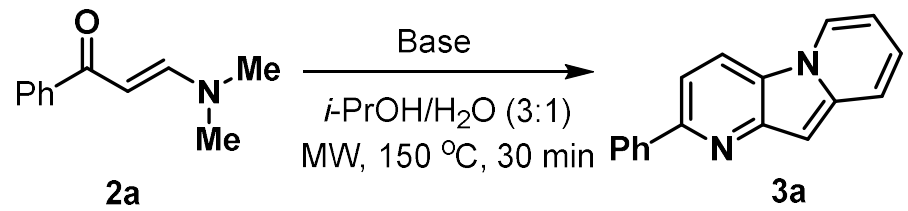

\begin{tabular}{|c|c|c|c|}
\hline Entry $^{a}$ & Base & 1a: 2a: Base & Yield, $\%$ b \\
\hline 1 & \multirow{6}{*}{$\mathrm{NaOAc}$} & $3: 1: 5$ & 34 \\
\hline 2 & & 3: $1: 1$ & 40 \\
\hline 3 & & 3: $1: 0.5$ & 46 \\
\hline 4 & & 3: 1: 0.1 & 12 \\
\hline 5 & & 1.5: $1: 0.5$ & 50 \\
\hline 6 & & 1: $1: 0.5$ & 44 \\
\hline 7 & $\mathrm{Et}_{3} \mathrm{~N}$ & 1.5: $1: 0.5$ & 31 \\
\hline 8 & DIPEA & 1.5: $1: 0.5$ & 25 \\
\hline 9 & $\mathrm{NH}_{4} \mathrm{OAc}$ & 1.5: $1: 0.5$ & 34 \\
\hline 10 & $\mathrm{~K}_{2} \mathrm{CO}_{3}$ & 1.5: $1: 0.5$ & 5 \\
\hline 11 & $\mathrm{Cs}_{2} \mathrm{CO}_{3}$ & 1.5: $1: 0.5$ & 21 \\
\hline $12^{c}$ & $\mathrm{NaOAc}$ & 1.5: $1: 0.5$ & 47 \\
\hline $13^{d}$ & $\mathrm{NaOAc}$ & 1.5: $1: 0.5$ & 33 \\
\hline $14^{\mathrm{e}}$ & $\mathrm{NaOAc}$ & 1.5: $1: 0.5$ & 37 \\
\hline
\end{tabular}

a A mixture of pyridinium salt $\mathbf{1}(0.591 \mathrm{mmol})$, enaminone 2 and the corresponding base in isopropyl alcohol (3 $\mathrm{mL})$ and water $(1 \mathrm{~mL})$ was irradiated in a closed vessel in a microwave reactor Monowave 300 (Anton Paar $\mathrm{GmbH})$ at $150{ }^{\circ} \mathrm{C}$ for $30 \mathrm{~min} .{ }^{\mathrm{b}}$ Isolated yield. ${ }^{\mathrm{c}}$ The reaction time was prolonged from 30 to $60 \mathrm{~min}$. ${ }^{\mathrm{d}}$ The reaction temperature was $120^{\circ} \mathrm{C}$. ${ }^{\mathrm{e}}$ The reaction temperature was $180^{\circ} \mathrm{C}$. 
With the optimized conditions in hand, we went on to investigate the reaction scope. It turned out that the reaction of $N$-cyanomethyl-2,3-dimethylpyridinium salt with enaminone was more effective, and the target product $3 \mathbf{b}$ was isolated with $82 \%$ yield (Scheme 2 ). On the contrary, the interaction of 2,5-dimethylpyridinium salt with the enaminone delivered product $3 \mathrm{c}$ with $27 \%$ yield. When p-methylphenyl-substituted enaminone was used, the compounds 3d-f were isolated with $21-37 \%$ yield. Bromo-substituted enaminones could be also used with various pyridinium salts to give

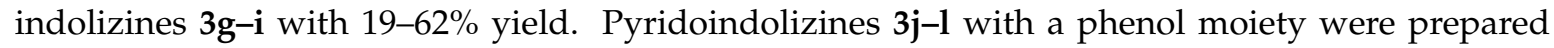
with 24-66\% yield. Moreover, we were pleased to find the pyridyl-containing enaminones to work successfully, producing the corresponding compounds $3 \mathrm{~m}-\mathbf{r}$ with poor to moderate yields. It is worth noting that taking $N$-cyanomethyl-2,3-dimethylpyridinium bromide in a large excess increased the yield of the compound 3n from 33\% to 85\%. Unfortunately, increasing the loading of the pyridinium salts in other cases did not result in yield improvement. The use of aliphatic enaminones $\left(\mathrm{R}^{3}=\mathrm{Me}\right.$ or $\mathrm{Et}$ ) in the reactions with $\mathrm{N}$-cyanomethyl-2,3-dimethylpyridinium bromide generated complex mixtures, and no target product could be isolated. As a rule, the use of 2,3-dimethylpyridiniums resulted in greater yields of the target pyridoindolizines 3 . The scope of the enaminones included various aryl groups, even phenols and heterocycles, while the use of aliphatic enaminones was found to be a limitation of the method.

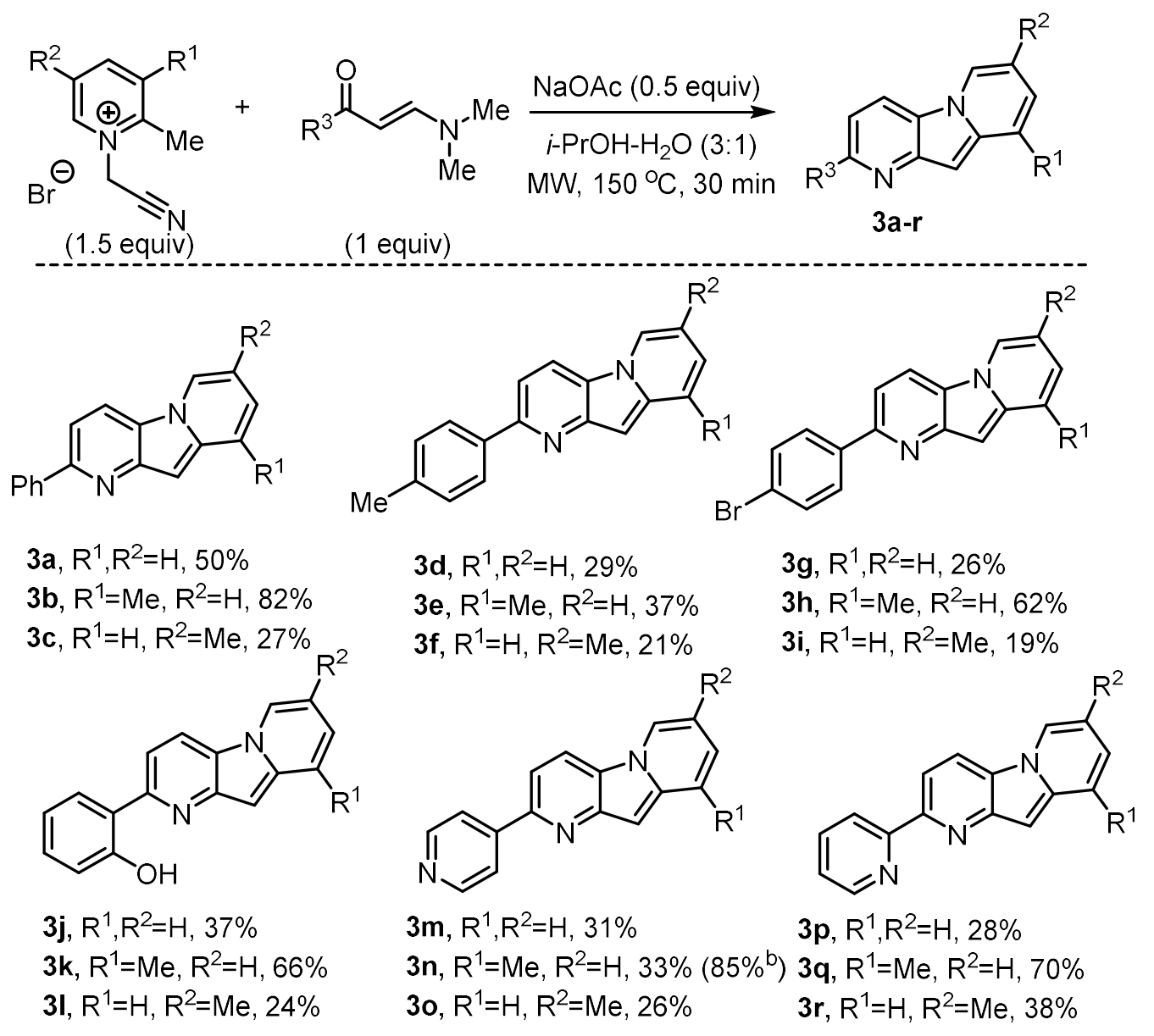

Scheme 2. The scope of the reaction between $N$-cyanomethyl-2-methylpyridinium bromides and various enaminones ${ }^{\text {a }}$ a General conditions: a mixture of pyridinium salt $\mathbf{1}$ (0.591 mmol), enaminone $2(0.394 \mathrm{mmol})$, and sodium acetate $(0.197 \mathrm{mmol})$ in isopropyl alcohol $(3 \mathrm{~mL})$ and water $(1 \mathrm{~mL})$ was placed into the microwave reactor and irradiated at $150{ }^{\circ} \mathrm{C}$ for $30 \mathrm{~min} .{ }^{\mathrm{b}}$ The reaction was performed on $1.182 \mathrm{mmol}$ scale of $\mathrm{N}$-cyanomethyl-2,3-dimethylpyridinium salt. 
The structure of pyridoindolizine $3 \mathbf{b}$ was unambiguously determined by a single crystal $\mathrm{X}$-ray diffraction study (Figure 1, CCDC 1922817).

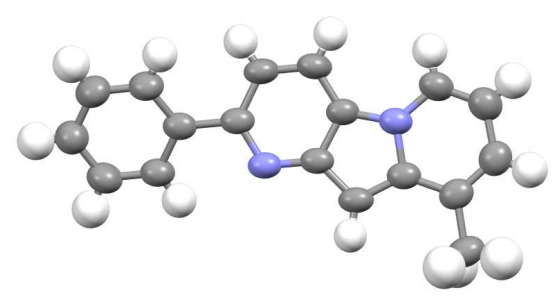

Figure 1. Crystal structure of 3b (CCDC 1922817).

The reaction presumably starts with the intramolecular cyclization of a deprotonated $\alpha$-methyl group on a nitrile moiety, eventually giving an aminoindolizine intermediate $\mathbf{A}$ [27] (Scheme 3). The interaction of the latter with enaminone produces an intermediate $\mathbf{B}$. The cyclocondensation of $\mathbf{B}$ completes the reaction sequence, delivering pyridoindolizine 3a. The intermediate $\mathbf{A}$ is evidently a highly nucleophilic species, containing a $\pi$-extensive pyrrole fragment combined with an amino group, readily reacting with the present electrophiles. Unfortunately, our attempts to isolate this intermediate failed. Even experiments in the absence of the enaminone generated multicomponent mixtures, pointing out the possibility for $\mathbf{A}$ to interact with the starting salt 1a.

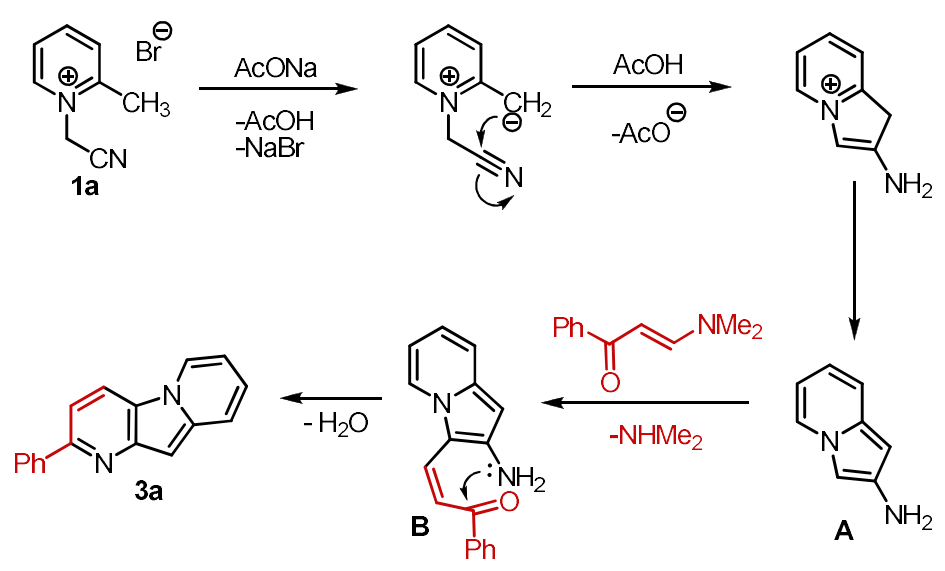

Scheme 3. Proposed mechanism for pyrido[2,3-b]indolizine formation.

The optical properties of the synthesized compounds were evaluated and all the spectra were measured in toluene solutions (Figure 2, Table 2, separate images are available in Supplementary Materials). Indolizines $3 \mathbf{a}-\mathbf{c}, \mathbf{m}-\mathbf{q}$ exhibited absorption peak maxima at $403-420 \mathrm{~nm}$. The emission peak maxima lay in the green region 505-528 nm, and the largest Stokes shift $4950 \mathrm{~cm}^{-1}$ was registered for compound $\mathbf{3 b}$. The fluorescence quantum yields (FQYs) were determined using coumarin 153 as a standard [32]. The lowest FQY values of 55-63\% were measured for 4-pyridyl-substituted pyridoindolizines $\mathbf{3 m}-\mathbf{o}$, while the phenyl-substituted pyridoindolizine $3 \mathbf{b}$ demonstrated the highest FQY, 82\%. This optical behavior is in accordance with the literature. For instance, indolizines, condensed with isoindole [33], quinoline [34,35] or dihydropyrrole [36] cycles also emit in the blue to green region $410-556 \mathrm{~nm}$ with FQYs up to $77 \%$. 


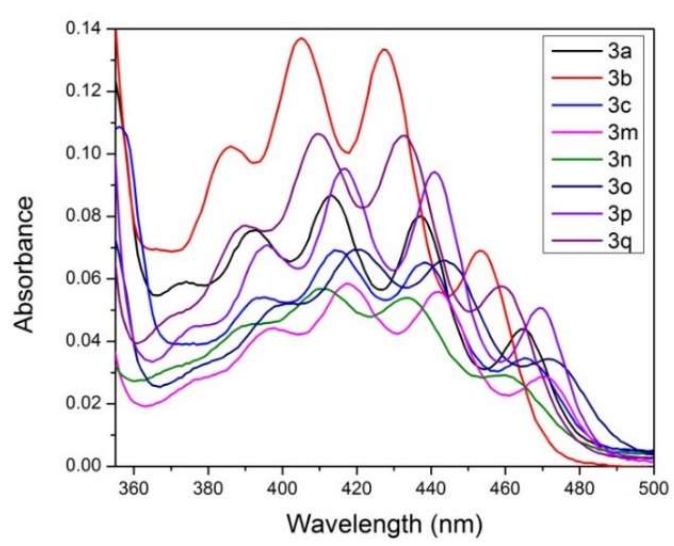

(a)

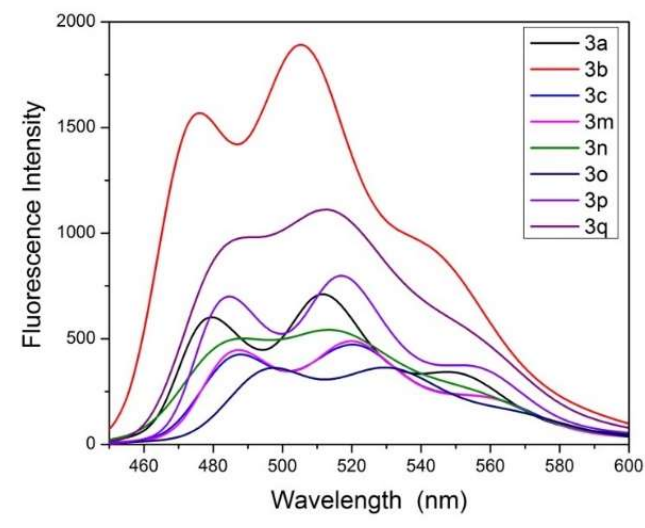

(b)

Figure 2. The absorbance (a) and emission (b) spectra of the above synthesized compounds in toluene.

Table 2. Photophysical properties of the synthesized compound.

\begin{tabular}{|c|c|c|c|c|c|}
\hline \multirow{2}{*}{ Compound } & Abs ${ }^{[a]}$ & $\varepsilon^{[b]}$ & Emission ${ }^{\text {[a] }}$ & FQY ${ }^{[c]}$ & Stokes Shift \\
\hline & {$[\mathrm{nm}]$} & {$\left[(\mathrm{M} \mathrm{cm})^{-1}\left(10^{9}\right)\right]$} & {$[\mathrm{nm}]$} & {$[\%]$} & {$\left[\mathrm{cm}^{-1}\right]$} \\
\hline $3 a$ & 413 & 1.652 & 511 & 77 & 4643 \\
\hline $3 b$ & 404 & 1.616 & 505 & 82 & 4950 \\
\hline $3 c$ & 414 & 1.656 & 520 & 64 & 4923 \\
\hline $3 m$ & 418 & 1.672 & 519 & 57 & 4655 \\
\hline $3 n$ & 410 & 1.640 & 513 & 63 & 4897 \\
\hline 30 & 420 & 1.680 & 528 & 55 & 4870 \\
\hline $3 p$ & 416 & 1.664 & 516 & 64 & 4658 \\
\hline $3 q$ & 409 & 1.636 & 512 & 77 & 4918 \\
\hline
\end{tabular}

\section{Materials and Methods}

\subsection{General Information}

Starting reagents were purchased from commercial sources and were used without any additional purification. Enaminones 2 were prepared according to the literature procedures [37]. Microwave reactions were conducted in a Monowave 300 Microwave Reactor (Anton Paar GmbH, Graz, Austria). Column chromatography was performed using silica gel (230-400 mesh) and mixtures in different proportions of ethyl acetate with hexane as the mobile phase. Melting points were determined on a SMP-10 apparatus (Barloworld Scientific Limited, Stone, UK). ${ }^{1} \mathrm{H}$ NMR spectra were recorded on a $400 \mathrm{MHz}$ spectrometer (Bruker, $100 \mathrm{MHz}$ for ${ }^{13} \mathrm{C} \mathrm{NMR}$ ) and referenced to the residual signals of the solvent (for ${ }^{1} \mathrm{H}$ and ${ }^{13} \mathrm{C}$ ). Chemical shifts are reported in parts per million ( $\left.\delta / \mathrm{ppm}\right)$. Coupling constants are reported in Hertz $(\mathrm{J} / \mathrm{Hz})$. The peak patterns are indicated as follows: $s$, singlet; $d$, doublet; $t$, triplet; q, quadruplet; $\mathrm{m}$, multiplet; $\mathrm{dd}$, doublet of doublets and td, triplet of doublets. Low resolution mass spectra were recorded with an LCMS-8040 triple quadrupole liquid chromatograph mass-spectrometer (Shimadzu corp., Tokyo, Japan). The reaction progress was monitored by TLC and the spots were visualized under UV light ( 254 or $365 \mathrm{~nm}$ ). Elemental analysis was performed on a EuroVector EA-3000 instrument (EuroVector S.p.A., Milan, Italy).

\subsection{General Procedure for the Synthesis of Salts $\mathbf{1 a - c}$}

Bromoacetonitrile $(0.026 \mathrm{~mol})$ was added to a stirred solution of corresponding pyridine $(0.022 \mathrm{~mol})$ in acetonitrile $(10 \mathrm{~mL})$. The reaction mixture was heated at reflux for $4 \mathrm{~h}$. The precipitate was filtered, washed with acetonitrile, and dried in vacuum over $\mathrm{P}_{2} \mathrm{O}_{5}$. 
N-(Cyanomethyl)-2-methylpyridinium bromide (1a). White powder; m.p. $195-196{ }^{\circ} \mathrm{C}$ (decomp.); yield, $3.56 \mathrm{~g}(76 \%) ;{ }^{1} \mathrm{H}$ NMR $\left(400 \mathrm{MHz}, \mathrm{CDCl}_{3}\right) \delta 9.16(1 \mathrm{H}, \mathrm{d}, J=6.1 \mathrm{~Hz}, \mathrm{H}-6), 8.59-8.62(1 \mathrm{H}, \mathrm{m}, \mathrm{H}-4), 8.16$ $(1 \mathrm{H}, \mathrm{d}, J=7.6 \mathrm{~Hz}, \mathrm{H}-3), 8.06-8.08(1 \mathrm{H}, \mathrm{m}, \mathrm{H}-5), 6.11\left(2 \mathrm{H}, \mathrm{d}, J=1.5 \mathrm{~Hz}, \mathrm{CH}_{2}-\mathrm{CN}\right), 2.90\left(3 \mathrm{H}, \mathrm{s}, \mathrm{C}_{2}-\mathrm{CH}_{3}\right)$. ${ }^{13} \mathrm{C}$ NMR $\left(100 \mathrm{MHz}, \mathrm{CDCl}_{3}\right) \delta 156.2,147.3,146.1,130.2,126.1,113.5,45.1,20.0$. ESI MS: $\mathrm{m} / z 133[\mathrm{M}]^{+}$. Elemental analysis calcd (\%) for $\mathrm{C}_{8} \mathrm{H}_{9} \mathrm{BrN}_{2}$ : C 45.10; $\mathrm{H} 4.26$; N 13.15; found: $\mathrm{C} 45.02 ; \mathrm{H}$ 4.29; N 13.26.

$\mathrm{N}$-(Cyanomethyl)-2,3-dimethylpyridinium bromide (1b). Light beige powder; m.p. $175-177^{\circ} \mathrm{C}$ (decomp.); yield, $3.60 \mathrm{~g}(72 \%)$; IR $\left(\mathrm{cm}^{-1}\right)$ : $2256(\mathrm{CN}) ;{ }^{1} \mathrm{H} \mathrm{NMR}\left(400 \mathrm{MHz}, \mathrm{CDCl}_{3}\right) \delta 9.05(1 \mathrm{H}, \mathrm{d}, J=6.1 \mathrm{~Hz}, \mathrm{H}-6)$, $8.53(1 \mathrm{H}, \mathrm{d}, J=8.1 \mathrm{~Hz}, \mathrm{H}-4), 7.98-8.01(1 \mathrm{H}, \mathrm{m}, \mathrm{H}-5), 6.20\left(2 \mathrm{H}, \mathrm{s}, \mathrm{CH}_{2}-\mathrm{CN}\right), 2.83\left(3 \mathrm{H}, \mathrm{s}, \mathrm{C}_{2}-\mathrm{CH}_{3}\right), 2.52$ $\left(3 \mathrm{H}, \mathrm{s}, \mathrm{C}_{3}-\mathrm{CH}_{3}\right) .{ }^{13} \mathrm{C}$ NMR $\left(100 \mathrm{MHz}, \mathrm{CDCl}_{3}\right) \delta 155.5,147.3,143.9,139.2,125.1,113.7,45.9,19.3,17.3$. ESI MS: $m / z 147[\mathrm{M}]^{+}$. Elemental analysis calcd (\%) for $\mathrm{C}_{9} \mathrm{H}_{11} \mathrm{BrN}_{2}$ : C 47.60; $\mathrm{H} 4.88 ; \mathrm{N} 12.34$; found: $\mathrm{C}$ 47.56; H 4.87; N 12.43.

$\mathrm{N}$-(Cyanomethyl)-2,5-dimethylpyridinium bromide (1c). Light beige powder; m.p. $168-169{ }^{\circ} \mathrm{C}$ (decomp.); yield, $2.90 \mathrm{~g}(58 \%) ;{ }^{1} \mathrm{H}$ NMR $\left(400 \mathrm{MHz}, \mathrm{CDCl}_{3}\right) \delta 9.11(1 \mathrm{H}, \mathrm{s}, \mathrm{H}-6), 8.49(1 \mathrm{H}, \mathrm{d}, J=8.3 \mathrm{~Hz}, \mathrm{H}-3), 8.08$ $(1 \mathrm{H}, \mathrm{d}, J=8.3 \mathrm{~Hz}, \mathrm{H}-4), 6.07\left(2 \mathrm{H}, \mathrm{s}, \mathrm{CH}_{2}-\mathrm{CN}\right), 2.88\left(3 \mathrm{H}, \mathrm{s}, \mathrm{C}_{2}-\mathrm{CH}_{3}\right), 2.46\left(3 \mathrm{H}, \mathrm{s}, \mathrm{C}_{5}-\mathrm{CH}_{3}\right) .{ }^{13} \mathrm{C} \mathrm{NMR}$ $\left(100 \mathrm{MHz}, \mathrm{CDCl}_{3}\right) \delta 153.2,147.9,145.3,136.5,129.5,113.5,44.9,19.4,17.4$. ESI MS: $m / z 147[\mathrm{M}]^{+}$. Elemental analysis calcd (\%) for $\mathrm{C}_{9} \mathrm{H}_{11} \mathrm{BrN}_{2}$ : C 47.60; $\mathrm{H} 4.88 ; \mathrm{N} 12.34$; found: $\mathrm{C} 47.57 ; \mathrm{H} 4.91 ; \mathrm{N} 12.45$.

\subsection{General Procedure for the Synthesis of Enaminones 2}

A mixture of dimethylformamide dimethylacetal $(14.8 \mathrm{mmol})$ and methyl ketone $(14.8 \mathrm{mmoL})$ was placed into the microwave reactor and irradiated at $150{ }^{\circ} \mathrm{C}$ for $15 \mathrm{~min}$, then left to cool to room temperature. After cooling, the precipitate was filtered-off, washed twice with toluene and dried on air. (E)-3-(Dimethylamino)-1-phenylprop-2-en-1-one (2a). Yellow powder; m.p. $91-92{ }^{\circ} \mathrm{C}$; yield, $1.17 \mathrm{~g}(45 \%)$;

${ }^{1} \mathrm{H}$ NMR $\left(400 \mathrm{MHz}, \mathrm{CDCl}_{3}\right) \delta 7.88(2 \mathrm{H}, \mathrm{d}, J=6.9 \mathrm{~Hz}, \mathrm{Ph}-\mathrm{H}), 7.78\left(1 \mathrm{H}, \mathrm{d}, J=12.4 \mathrm{~Hz}, \mathrm{CH}=\mathrm{CH}-\mathrm{NMe}_{2}\right)$, 7.39-7.45 (3H, m, Ph-H), $5.70\left(1 \mathrm{H}, \mathrm{d}, J=12.4 \mathrm{~Hz}, \mathrm{CH}=\mathrm{CH}-\mathrm{NMe}_{2}\right), 3.11\left(3 \mathrm{H}, \mathrm{s}, \mathrm{N}_{-} \mathrm{CH}_{3}\right), 2.90(3 \mathrm{H}, \mathrm{s}$, $\left.\mathrm{N}-\mathrm{CH}_{3}\right) .{ }^{13} \mathrm{C} \mathrm{NMR}\left(100 \mathrm{MHz}, \mathrm{CDCl}_{3}\right) \delta 188.8,154.4,140.7,131.0,128.2(2 \mathrm{C}), 127.6$ (2C), 92.3, 45.1, 37.4. ESI MS: $m / z 176[\mathrm{M}+\mathrm{H}]^{+}$.

(E)-3-(Dimethylamino)-1-(p-tolyl)prop-2-en-1-one (2b). Yellow powder; m.p. 88-89 ${ }^{\circ} \mathrm{C}$; yield, $0.62 \mathrm{~g} \mathrm{(24 \% )}$; ${ }^{1} \mathrm{H}$ NMR $\left(400 \mathrm{MHz} \mathrm{CDCl}_{3}\right) \delta 7.80(2 \mathrm{H}, \mathrm{d}, J=8.1 \mathrm{~Hz}, \mathrm{Ph}-\mathrm{H}), 7.77\left(1 \mathrm{H}, \mathrm{d}, J=12.3 \mathrm{~Hz}, \mathrm{CH}=\mathrm{CH}-\mathrm{NMe}_{2}\right)$, $7.19(2 \mathrm{H}, \mathrm{d}, J=8.1 \mathrm{~Hz}, \mathrm{Ph}-\mathrm{H}), 5.70\left(1 \mathrm{H}, \mathrm{d}, J=12.3 \mathrm{~Hz}, \mathrm{CH}=\mathrm{CH}-\mathrm{NMe}_{2}\right), 3.09\left(3 \mathrm{H}, \mathrm{s}, \mathrm{N}-\mathrm{CH}_{3}\right), 2.89(3 \mathrm{H}, \mathrm{s}$, $\left.\mathrm{N}-\mathrm{CH}_{3}\right), 2.37\left(3 \mathrm{H}, \mathrm{s}, \mathrm{Ph}-\mathrm{CH}_{3}\right) .{ }^{13} \mathrm{C} \mathrm{NMR}\left(100 \mathrm{MHz}, \mathrm{CDCl}_{3}\right) \delta 188.2,153.9,141.1,137.7,128.7(2 \mathrm{C}), 127.5$ (2C), 92.0, 44.8, 37.1, 21.3. ESI MS: $m / z 190[\mathrm{M}+\mathrm{H}]^{+}$.

(E)-3-(Dimethylamino)-1-(2-hydroxyphenyl)prop-2-en-1-one (2d). Orange powder; m.p. 133-135 ${ }^{\circ} \mathrm{C}$; yield, $1.58 \mathrm{~g}(56 \%) ;{ }^{1} \mathrm{H} \mathrm{NMR}\left(400 \mathrm{MHz}, \mathrm{CDCl}_{3}\right) \delta 13.98\left(1 \mathrm{H}, \mathrm{s}, \mathrm{C}_{2}-\mathrm{OH}\right), 7.87\left(1 \mathrm{H}, \mathrm{d}, J=12.3 \mathrm{~Hz}, \mathrm{CH}=\mathrm{CH}-\mathrm{NMe}_{2}\right)$, $7.69(1 \mathrm{H}, \mathrm{dd}, J=8.1,1.1 \mathrm{~Hz}, \mathrm{Ph}-\mathrm{H}), 7.35(1 \mathrm{H}, \mathrm{m}, \mathrm{Ph}-\mathrm{H}), 6.93(1 \mathrm{H}, \mathrm{d}, J=8.1, \mathrm{Ph}-\mathrm{H}), 6.80(1 \mathrm{H}, \mathrm{m}, \mathrm{Ph}-\mathrm{H})$, $5.77\left(1 \mathrm{H}, \mathrm{d}, J=12.3 \mathrm{~Hz}, \mathrm{CH}=\mathrm{CH}-\mathrm{NMe}_{2}\right), 3.18\left(3 \mathrm{H}, \mathrm{s}, \mathrm{N}-\mathrm{CH}_{3}\right), 2.96\left(3 \mathrm{H}, \mathrm{s}, \mathrm{N}-\mathrm{CH}_{3}\right) .{ }^{13} \mathrm{C} \mathrm{NMR}(100 \mathrm{MHz}$, $\left.\mathrm{CDCl}_{3}\right) \delta 191.6,163.0,154.9,134.0,128.3,120.4,118.3,118.1,90.1,45.5,37.5$. ESI MS: $m / z 192[\mathrm{M}+\mathrm{H}]^{+}$.

(E)-3-(Dimethylamino)-1-(pyridin-4-yl)prop-2-en-1-one (2e). Yellow powder; m.p. $115-116{ }^{\circ} \mathrm{C}$; yield, $0.886 \mathrm{~g}(34 \%) ;{ }^{1} \mathrm{H}$ NMR $\left(400 \mathrm{MHz}, \mathrm{CDCl}_{3}\right) \delta 8.67(2 \mathrm{H}, \mathrm{d}, J=5.6, \mathrm{Ar}-\mathrm{H}), 7.82(1 \mathrm{H}, \mathrm{d}, J=11.9 \mathrm{~Hz}$, $\left.\mathrm{CH}=\mathrm{CH}-\mathrm{NMe}_{2}\right), 7.67(2 \mathrm{H}, \mathrm{d}, J=5.6 \mathrm{~Hz}, \mathrm{Ar}-\mathrm{H}), 5.63\left(1 \mathrm{H}, \mathrm{d}, J=11.9 \mathrm{~Hz}, \mathrm{CH}=\mathrm{CH}-\mathrm{NMe}_{2}\right), 3.16(3 \mathrm{H}, \mathrm{s}$, $\left.\mathrm{N}-\mathrm{CH}_{3}\right), 2.93\left(3 \mathrm{H}, \mathrm{s}, \mathrm{N}-\mathrm{CH}_{3}\right) .{ }^{13} \mathrm{C} \mathrm{NMR}\left(100 \mathrm{MHz}, \mathrm{CDCl}_{3}\right) \delta 186.4,155.2,149.9(2 \mathrm{C}), 147.3,121.2(2 \mathrm{C})$, 91.6, 45.2, 37.4. ESI MS: $m / z 177[\mathrm{M}+\mathrm{H}]^{+}$.

\subsection{General Procedure for the Synthesis of Compounds $3 \boldsymbol{a}-\boldsymbol{r}$}

Method A (for 3a-g, 3i, 3m-r): A mixture of pyridinium salt $\mathbf{1}$ (0.591 mmol), enaminone 2 $(0.394 \mathrm{mmol})$, and sodium acetate $(0.197 \mathrm{mmol})$ in isopropyl alcohol $(3 \mathrm{~mL})$ and water $(1 \mathrm{~mL})$ was placed into the microwave reactor and irradiated at $150^{\circ} \mathrm{C}$ for $30 \mathrm{~min}$. After cooling to room temperature, 
the solvent was then evaporated under reduced pressure. The products were isolated by column chromatography on silica gel, eluting with ethyl acetate-hexane mixture in different proportions.

Method B (for 3h, 3k): A mixture of pyridinium salt 1 ( $0.591 \mathrm{mmol})$, enaminone $2(0.394 \mathrm{mmol})$, and sodium acetate $(0.197 \mathrm{mmol})$ in isopropyl alcohol $(3 \mathrm{~mL})$ and water $(1 \mathrm{~mL})$ was placed into the microwave reactor and irradiated at $150{ }^{\circ} \mathrm{C}$ for $30 \mathrm{~min}$ and left to cool to room temperature. After cooling, the precipitate was filtered-off and washed with ethanol, water ( 2 times) and ethanol again, then dried in air.

Method C (for 3j, 31): A mixture of pyridinium salt 1 (0.591 mmol), enaminone 2 (0.394 mmol), and sodium acetate $(0.197 \mathrm{mmol})$ in isopropyl alcohol $(3 \mathrm{~mL})$ and water $(1 \mathrm{~mL})$ was placed into the microwave reactor and irradiated at $150^{\circ} \mathrm{C}$ for $30 \mathrm{~min}$ and left to cool to room temperature. The reaction mixture was diluted with water $(70 \mathrm{~mL})$ and extracted with DCM. The combined organic layer was dried over $\mathrm{Na}_{2} \mathrm{SO}_{4}$. After filtration, the solvent was evaporated under reduced pressure. The residue was recrystallized from the isopropyl alcohol-DCM 3-1 mixture. The precipitate was filtered-off and washed with isopropyl alcohol for 3 times, then dried in air.

2-Phenylpyrido[2,3-b]indolizine (3a). Prepared according to the Method A. Eluent ethyl acetate-hexane 1: 10. Light brown powder; m.p. 178-180 ${ }^{\circ} \mathrm{C}$ (decomp.); yield, $48 \mathrm{mg}(50 \%) ;{ }^{1} \mathrm{H} \mathrm{NMR}\left(400 \mathrm{MHz}, \mathrm{CDCl}_{3}\right) \delta 8.25$ $(\mathrm{d}, J=7.1 \mathrm{~Hz}, 1 \mathrm{H}, \mathrm{H}-6), 8.15(\mathrm{~d}, J=8.6 \mathrm{~Hz}, 1 \mathrm{H}, \mathrm{H}-3), 8.11(\mathrm{~d}, J=7.6 \mathrm{~Hz}, 2 \mathrm{H}, \mathrm{Ph}-\mathrm{H}), 7.61(\mathrm{~d}, J=8.6 \mathrm{~Hz}, 1 \mathrm{H}$, H-4), 7.50-7.52 (m, 3H, H-9, Ph-H), 7.42-7.44 (m, 1H, Ph-H), 6.95-6.98 (m, 1H, H-7), 6.90 (s, 1H, H-10), 6.53-6.55 (m, 1H, H-8). ${ }^{13} \mathrm{C}$ NMR (100 MHz, $\left.\mathrm{CDCl}_{3}\right) \delta 154.9,146.3,140.7,139.2,128.6(2 \mathrm{C}), 128.4,127.5$ (2C), 124.6, 123.4, 121.7, 119.4, 118.0, 112.2, 108.7, 92.5. ESI MS: $m / z 245[\mathrm{M}+\mathrm{H}]^{+}$. Elemental analysis calcd (\%) for $\mathrm{C}_{17} \mathrm{H}_{12} \mathrm{~N}_{2}$ : C 83.58; $\mathrm{H} 4.95 ; \mathrm{N}$ 11.47; found: C 83.54; $\mathrm{H} 4.98 ; \mathrm{N} 11.59$.

9-Methyl-2-phenylpyrido[2,3-b]indolizine (3b). Prepared according to the Method A. Eluent ethyl acetate-hexane 1: 5. Yellow powder; m.p. ${ }^{143-146}{ }^{\circ} \mathrm{C}$ (decomp.); yield, $83 \mathrm{mg}(82 \%) ;{ }^{1} \mathrm{H}$ NMR $\left(400 \mathrm{MHz}, \mathrm{CDCl}_{3}\right) \delta 8.16-8.20(\mathrm{~m}, 2 \mathrm{H}, \mathrm{H}-3, \mathrm{H}-6), 8.12(\mathrm{~d}, J=7.6 \mathrm{~Hz}, 2 \mathrm{H}, \mathrm{Ph}-\mathrm{H}), 7.62(\mathrm{~d}, J=8.6 \mathrm{~Hz}, 1 \mathrm{H}$, $\mathrm{H}-4), 7.50-7.53$ (m, 2H, Ph-H), 7.41-7.44 (m, 1H,, Ph-H), 6.88 (s, 1H, H-10), 6.80 (d, J = 6.1 Hz, 1H, H-8), 6.52-6.55 (m, 1H, H-7), 2.51 (s, 3H, $\left.\mathrm{C}_{9}-\mathrm{CH}_{3}\right) .{ }^{13} \mathrm{C} \mathrm{NMR}\left(100 \mathrm{MHz}, \mathrm{CDCl}_{3}\right) \delta$ 154.7, 146.2, 140.6 (2C), 128.7 (3C), 128.4, 127.5 (2C), 122.3 (2C), 122.0, 118.3, 112.2, 109.0, 91.1, 18.5. ESI MS: $m / z 259[\mathrm{M}+\mathrm{H}]^{+}$. Elemental analysis calcd (\%) for $\mathrm{C}_{18} \mathrm{H}_{14} \mathrm{~N}_{2}$ : C 83.69; H 5.46; N 10.84; found: C 83.66; H 5.51; N 10.95.

7-Methyl-2-phenylpyrido[2,3-b]indolizine (3c). Prepared according to the Method A. Eluent ethyl acetate-hexane 1: 5. Dark yellow powder; m.p. $175-176{ }^{\circ} \mathrm{C}$ (decomp.); yield, $27 \mathrm{mg}(27 \%) ;{ }^{1} \mathrm{H} \mathrm{NMR}$ $\left(400 \mathrm{MHz}, \mathrm{CDCl}_{3}\right) \delta 8.15(\mathrm{~d}, J=8.6 \mathrm{~Hz}, 1 \mathrm{H}, \mathrm{H}-3), 8.10(\mathrm{~d}, J=7.6 \mathrm{~Hz}, 2 \mathrm{H}, \mathrm{Ph}-\mathrm{H}), 8.08(\mathrm{~s}, 1 \mathrm{H}, \mathrm{H}-6), 7.59$ (d, J = 8.6 Hz, 1H, H-4), 7.49-7.52 (m, 2H, Ph-H), 7.41-7.46 (m, 2H, H-9, Ph-H), 6.86-6.87 (m, 2H, H-8, $\mathrm{H}-10), 2.31\left(3 \mathrm{H}, \mathrm{c}, \mathrm{C}_{7}-\mathrm{CH}_{3}\right) .{ }^{13} \mathrm{C} \mathrm{NMR}\left(100 \mathrm{MHz}, \mathrm{CDCl}_{3}\right) \delta 154.5,146.0,140.7,138.3,128.6$ (2C), 128.3, $127.5(2 \mathrm{C}), 127.1,121.8,121.6,119.0,118.1(2 \mathrm{C}), 112.0,91.9,18.3$. ESI MS: $m / z 259[\mathrm{M}+\mathrm{H}]^{+}$. Elemental analysis calcd (\%) for $\mathrm{C}_{18} \mathrm{H}_{14} \mathrm{~N}_{2}$ : C 83.69; $\mathrm{H} 5.46 ; \mathrm{N} 10.84$; found: C 83.62; $\mathrm{H}$ 5.48; $\mathrm{N} 10.99$.

2-(p-Tolyl)pyrido[2,3-b]indolizine (3d). Prepared according to the Method A. Eluent ethyl acetate-hexane 1: 10. Bright yellow powder; m.p. $181-182{ }^{\circ} \mathrm{C}$ (decomp.); yield, $29 \mathrm{mg}(29 \%) ;{ }^{1} \mathrm{H} \mathrm{NMR}(400 \mathrm{MHz}$, $\left.\mathrm{CDCl}_{3}\right) \delta 8.90(\mathrm{~d}, J=7.1 \mathrm{~Hz}, 1 \mathrm{H}, \mathrm{H}-6), 8.67(\mathrm{~d}, J=8.6 \mathrm{~Hz}, 1 \mathrm{H}, \mathrm{H}-3), 8.09(\mathrm{~d}, J=8.1 \mathrm{~Hz}, 2 \mathrm{H}, \mathrm{Ph}-\mathrm{H}), 7.80$ (d, $J=8.6 \mathrm{~Hz}, 1 \mathrm{H}, \mathrm{H}-4), 7.61$ (d, $J=9.6 \mathrm{~Hz}, 1 \mathrm{H}, \mathrm{H}-9), 7.30$ (d, $J=8.1 \mathrm{~Hz}, 2 \mathrm{H}, \mathrm{Ph}-\mathrm{H}), 7.09-7.11(\mathrm{~m}, 1 \mathrm{H}$, $\mathrm{H}-7), 6.78$ (s, 1H, H-10), 6.68-6.70 (m, 1H, H-8), 2.37 (s, 3H, Ph-CH $\left.{ }_{3}\right) .{ }^{13} \mathrm{C}$ NMR $\left(100 \mathrm{MHz}, \mathrm{CDCl}_{3}\right) \delta$ 153.2, 145.4, 138.7, 137.9, 137.2, 129.3 (2C), 126.8 (2C), 126.3, 124.1, 121.5, 119.6, 118.7, 111.3, 108.8, 91.5, 20.8. ESI MS: $m / z 259[\mathrm{M}+\mathrm{H}]^{+}$. Elemental analysis calcd (\%) for $\mathrm{C}_{18} \mathrm{H}_{14} \mathrm{~N}_{2}: \mathrm{C}, 83.69 ; \mathrm{H}, 5.46 ; \mathrm{N}, 10.84$; found: C 83.65; H 5.49; N 10.96 .

9-Methyl-2-(p-tolyl)pyrido[2,3-b]indolizine (3e). Prepared according to the Method A. Eluent ethyl acetate-hexane 1: 15. Yellow powder; m.p. $174{ }^{\circ} \mathrm{C}$ (decomp.); yield, $87 \mathrm{mg}(81 \%) ;{ }^{1} \mathrm{H} \mathrm{NMR}(400 \mathrm{MHz}$, $\left.\mathrm{CDCl}_{3}\right) \delta 8.79(\mathrm{~d}, J=6.6 \mathrm{~Hz}, 1 \mathrm{H}, \mathrm{H}-6), 8.66(\mathrm{~d}, J=8.6 \mathrm{~Hz}, 1 \mathrm{H}, \mathrm{H}-3), 8.09(\mathrm{~d}, J=8.1 \mathrm{~Hz}, 2 \mathrm{H}, \mathrm{Ph}-\mathrm{H}), 7.80$ $(\mathrm{d}, J=8.6 \mathrm{~Hz}, 1 \mathrm{H}, \mathrm{H}-4), 7.31(\mathrm{~d}, J=7.6 \mathrm{~Hz}, 2 \mathrm{H}, \mathrm{Ph}-\mathrm{H}), 6.93(\mathrm{~d}, J=6.1 \mathrm{~Hz}, 1 \mathrm{H}, \mathrm{H}-8), 6.77(\mathrm{~s}, 1 \mathrm{H}, \mathrm{H}-10)$, 6.65-6.67 (m, 1H, H-7), $2.46\left(\mathrm{~s}, 3 \mathrm{H}, \mathrm{C}_{9}-\mathrm{CH}_{3}\right), 2.37$ (s, 3H, Ph-CH $\mathrm{CH}_{3} .{ }^{13} \mathrm{C} \mathrm{NMR}\left(100 \mathrm{MHz}, \mathrm{CDCl}_{3}\right) \delta$ 153.1, 
145.4, 139.7, 137.8, 137.2, 129.2 (2C), 127.2, 126.8 (2C), 123.9, 122.5, 122.1, 119.8, 111.4, 108.9, 90.3, 20.8, 18.0. ESI MS: $m / z 273[\mathrm{M}+\mathrm{H}]^{+}$. Elemental analysis calcd (\%) for $\mathrm{C}_{19} \mathrm{H}_{16} \mathrm{~N}_{2}$ : $\mathrm{C}, 83.79 ; \mathrm{H}, 5.92 ; \mathrm{N}, 10.29$; found: C 83.72; H 5.89; N 10.43 .

7-Methyl-2-(p-tolyl)pyrido[2,3-b]indolizine (3f). Prepared according to the Method A. Eluent ethyl acetate-hexane 1: 15. Dark yellow powder; m.p. $173-174{ }^{\circ} \mathrm{C}$ (decomp.); yield, $23 \mathrm{mg}(21 \%) ;{ }^{1} \mathrm{H}$ NMR $\left(400 \mathrm{MHz}, \mathrm{CDCl}_{3}\right) \delta 8.73(\mathrm{~s}, 1 \mathrm{H}, \mathrm{H}-6), 8.60(\mathrm{~d}, J=8.6 \mathrm{~Hz}, 1 \mathrm{H}, \mathrm{H}-3), 8.08(\mathrm{~d}, J=8.1 \mathrm{~Hz}, 2 \mathrm{H}, \mathrm{Ph}-\mathrm{H}), 7.77$ $(\mathrm{d}, J=8.6 \mathrm{~Hz}, 1 \mathrm{H}, \mathrm{H}-4), 7.55(\mathrm{~d}, J=9.3,1 \mathrm{H}, \mathrm{H}-9), 7.30(\mathrm{~d}, J=7.6 \mathrm{~Hz}, 2 \mathrm{H}, \mathrm{Ph}-\mathrm{H}), 6.98(\mathrm{~d}, J=9.3 \mathrm{~Hz}, 1 \mathrm{H}$, $\mathrm{H}-8), 6.73$ (s, 1H, H-10), 2.37 (s, 3H, Ph- $\left.\mathrm{CH}_{3}\right), 2.28\left(\mathrm{~s}, 3 \mathrm{H}, \mathrm{C}_{7}-\mathrm{CH}_{3}\right) .{ }^{13} \mathrm{C} \mathrm{NMR}\left(100 \mathrm{MHz}, \mathrm{CDCl}_{3}\right) \delta 152.9$, 145.3, 137.8, 137.6, 137.2, 129.2 (2C), 127.5, 126.7 (2C), 123.2, 121.3, 119.4, 118.3, 117.7, 111.1, 91.0, 20.8, 17.7. ESI MS: $m / z 273[\mathrm{M}+\mathrm{H}]^{+}$. Elemental analysis calcd (\%) for $\mathrm{C}_{19} \mathrm{H}_{16} \mathrm{~N}_{2}$ : $\mathrm{C}, 83.79 ; \mathrm{H}, 5.92 ; \mathrm{N}, 10.29$; found: C 83.84; H 5.90; N 10.33 .

2-(4-Bromophenyl)pyrido[2,3-b]indolizine (3g). Prepared according to the Method A. Eluent ethyl acetate-hexane 1: 15 . Brown powder; m.p. $215-217{ }^{\circ} \mathrm{C}$ (decomp.); yield, $33 \mathrm{mg}(26 \%) ;{ }^{1} \mathrm{H} \mathrm{NMR}$ $\left(400 \mathrm{MHz}, \mathrm{CDCl}_{3}\right) \delta 8.93(\mathrm{~d}, J=7.1 \mathrm{~Hz}, 1 \mathrm{H}, \mathrm{H}-6), 8.72(\mathrm{~d}, J=9.1 \mathrm{~Hz}, 1 \mathrm{H}, \mathrm{H}-3), 8.15(\mathrm{~d}, J=8.6 \mathrm{~Hz}, 2 \mathrm{H}$, Ph-H), $7.86(\mathrm{~d}, J=9.1 \mathrm{~Hz}, 1 \mathrm{H}, \mathrm{H}-4), 7.69(\mathrm{~d}, J=8.6 \mathrm{~Hz}, 2 \mathrm{H}, \mathrm{Ph}-\mathrm{H}), 7.62(\mathrm{~d}, J=9.1 \mathrm{~Hz}, 1 \mathrm{H}, \mathrm{H}-9), 7.11-7.14$ (m, 1H, H-8), 6.80 (s, 1H, H-10), 6.70-6.73 (m, 1H, H-7). ${ }^{13} \mathrm{C}$ NMR (100 MHz, $\left.\mathrm{CDCl}_{3}\right) \delta 151.9,145.5$, 139.1, 139.0, 131.6 (2C), 128.9 (2C), 126.4, 124.5, 122.1, 121.8, 119.9, 118.7, 111.3, 108.9, 91.6. ESI MS: $\mathrm{m} / \mathrm{z}$ $324[\mathrm{M}+\mathrm{H}]^{+}$. Elemental analysis calcd (\%) for $\mathrm{C}_{17} \mathrm{H}_{11} \mathrm{BrN}_{2}$ : C 63.18; $\mathrm{H} 3.43 ; \mathrm{N}$ 8.67; found: $\mathrm{C} 63.25 ; \mathrm{H}$ 3.40; N 8.63.

2-(4-Bromophenyl)-9-methylpyrido[2,3-b]indolizine (3h). Prepared according to the Method B. Gold powder; m.p. $192-193{ }^{\circ} \mathrm{C}$ (decomp.); yield, $82 \mathrm{mg}(62 \%) ;{ }^{1} \mathrm{H} \mathrm{NMR}\left(400 \mathrm{MHz}, \mathrm{CDCl}_{3}\right) \delta 8.80$ (d, $J=7.1 \mathrm{~Hz}, 1 \mathrm{H}, \mathrm{H}-6), 8.70(\mathrm{~d}, J=8.6 \mathrm{~Hz}, 1 \mathrm{H}, \mathrm{H}-3), 8.16(\mathrm{~d}, J=8.3 \mathrm{~Hz}, 2 \mathrm{H}, \mathrm{Ph}-\mathrm{H}), 7.85(\mathrm{~d}, J=8.6 \mathrm{~Hz}, 1 \mathrm{H}$, H-4), $7.69(\mathrm{~d}, J=8.3 \mathrm{~Hz}, 2 \mathrm{H}, \mathrm{Ph}-\mathrm{H}), 6.95(\mathrm{~d}, J=6.6 \mathrm{~Hz}, 1 \mathrm{H}, \mathrm{H}-8), 6.79(\mathrm{~s}, 1 \mathrm{H}, \mathrm{H}-10), 6.66-6.69(\mathrm{~m}, 1 \mathrm{H}$, $\mathrm{H}-7), 2.46\left(\mathrm{~s}, 3 \mathrm{H}, \mathrm{C}_{9}-\mathrm{CH}_{3}\right) .{ }^{13} \mathrm{C}$ NMR $\left(100 \mathrm{MHz}, \mathrm{CDCl}_{3}\right) \delta 151.8,145.5,140.0,139.1,131.5(2 \mathrm{C}), 128.9$ (2C), 127.2, 124.0, 122.7, 122.4, 122.0, 119.9, 111.4, 109.0, 90.4, 18.0. ESI MS: $m / z 338[\mathrm{M}+\mathrm{H}]^{+}$. Elemental analysis calcd (\%) for $\mathrm{C}_{18} \mathrm{H}_{13} \mathrm{BrN}_{2}$ : C 64.11; H 3.89; $\mathrm{N}$ 8.31; found: C 64.01; H 3.91; $\mathrm{N}$ 8.36.

2-(4-Bromophenyl)-7-methylpyrido[2,3-b]indolizine (3i). Prepared according to the Method A. Eluent ethyl acetate-hexane 1: 15. Light brown powder; m.p. $177-178{ }^{\circ} \mathrm{C}$ (decomp.); yield, $25 \mathrm{mg}(19 \%) ;{ }^{1} \mathrm{H} \mathrm{NMR}$ $\left(400 \mathrm{MHz}, \mathrm{CDCl}_{3}\right) \delta 8.72(\mathrm{~s}, 1 \mathrm{H}, \mathrm{H}-6), 8.61$ (d, J = 8.1 Hz, 1H, H-3), 8.14 (d, J = 7.6 Hz, 2H, Ph-H), 7.79 $(\mathrm{d}, J=8.1 \mathrm{~Hz}, 1 \mathrm{H}, \mathrm{H}-4), 7.67$ (d, J = 7.6 Hz, 2H, Ph-H), 7.55 (d, J = 9.1 Hz, 1H, H-9), 6.99 (d, J = 9.1 Hz, $1 \mathrm{H}, \mathrm{H}-8), 6.74$ (s, $1 \mathrm{H}, \mathrm{H}-10), 2.27$ (s, 3H, $\left.\mathrm{C}_{7}-\mathrm{CH}_{3}\right) .{ }^{13} \mathrm{C} \mathrm{NMR}\left(100 \mathrm{MHz}, \mathrm{CDCl}_{3}\right) \delta 151.5,145.3,139.1$, 137.9, 131.5 (2C), 128.9 (2C), 127.7, 123.2, 121.9, 121.5, 119.5, 118.3, 117.9, 111.1, 91.1, 17.7. ESI MS: $\mathrm{m} / \mathrm{z}$ $338[\mathrm{M}+\mathrm{H}]^{+}$. Elemental analysis calcd (\%) for $\mathrm{C}_{18} \mathrm{H}_{13} \mathrm{BrN}_{2}$ : C 64.11; $\mathrm{H} 3.89 ; \mathrm{N}$ 8.31; found: $\mathrm{C} 64.17 ; \mathrm{H}$ $3.92 ; \mathrm{N} 8.34$.

2-(Pyrido[2,3-b]indolizin-2-yl)phenol (3j). Prepared according to the Method C. Dark brown powder; m.p. $230-232{ }^{\circ} \mathrm{C}$ (decomp.); yield, $38 \mathrm{mg}(37 \%) ;{ }^{1} \mathrm{H}$ NMR $\left(400 \mathrm{MHz}, \mathrm{CDCl}_{3}\right) \delta 15.09$ (s, $\left.1 \mathrm{H}, \mathrm{OH}\right), 8.99$ $(\mathrm{d}, J=6.6 \mathrm{~Hz}, 1 \mathrm{H}, \mathrm{H}-6), 8.85(\mathrm{~d}, J=8.9 \mathrm{~Hz}, 1 \mathrm{H}, \mathrm{H}-3), 8.13(\mathrm{~d}, J=7.6 \mathrm{~Hz}, 1 \mathrm{H}, \mathrm{Ph}-\mathrm{H}), 8.06(\mathrm{~d}, J=8.9 \mathrm{~Hz}$, $1 \mathrm{H}, \mathrm{H}-4), 7.65$ (d, J = 9.1 Hz, 1H, H-9), 7.29-7.31 (m, 1H, Ph-H), 7.18-7.20 (m, 1H, H-8), 6.92-6.96 (m, 2H, Ph-H), 6.84 (s, 1H, H-10), 6.78-6.80 (m, 1H, H-7). $\left.{ }^{13} \mathrm{C} \mathrm{NMR} \mathrm{(100} \mathrm{MHz,} \mathrm{CDCl}_{3}\right) \delta 159.5,154.4,141.6$, $139.3,130.8,127.3,126.3,125.1,121.6,121.5,119.7,118.7,118.6,117.8,110.2,109.5,90.3$. ESI MS: $m / z 261$ $[\mathrm{M}+\mathrm{H}]^{+}$. Elemental analysis calcd (\%) for $\mathrm{C}_{17} \mathrm{H}_{12} \mathrm{~N}_{2} \mathrm{O}: \mathrm{C}$ 78.44; $\mathrm{H} 4.65 ; \mathrm{N} 10.76$; found: $\mathrm{C} 78.39 ; \mathrm{H}$ $4.62 ; \mathrm{N} 10.82$.

2-(9-Methylpyrido[2,3-b]indolizin-2-yl)phenol (3k). Prepared according to the Method B. Gold powder; m.p. $179{ }^{\circ} \mathrm{C}$ (decomp.); yield, $71 \mathrm{mg}(66 \%) ;{ }^{1} \mathrm{H}$ NMR $\left(400 \mathrm{MHz}, \mathrm{CDCl}_{3}\right) \delta 15.10(\mathrm{~s}, 1 \mathrm{H}, \mathrm{OH}), 8.87(\mathrm{~d}$, $J=6.6 \mathrm{~Hz}, 1 \mathrm{H}, \mathrm{H}-6), 8.84(\mathrm{~d}, J=8.6 \mathrm{~Hz}, 1 \mathrm{H}, \mathrm{H}-3), 8.13(\mathrm{~d}, J=7.6 \mathrm{~Hz}, 1 \mathrm{H}, \mathrm{Ph}-\mathrm{H}), 8.06(\mathrm{~d}, J=8.6 \mathrm{~Hz}, 1 \mathrm{H}$, H-4), 7.29-7.31 (m, 1H, Ph-H), 7.01 (d, J = 6.6 Hz, 1H, H-8), 6.92-6.96 (m, 2H, Ph-H), $6.84(\mathrm{~s}, 1 \mathrm{H}, \mathrm{H}-10)$, 6.73-6.76 (m, 1H, H-7), 2.47 (s, 3H, $\left.\mathrm{C}_{9}-\mathrm{CH}_{3}\right) .{ }^{13} \mathrm{C} \mathrm{NMR}\left(100 \mathrm{MHz}, \mathrm{CDCl}_{3}\right) \delta 159.5,154.3,141.5,140.3$, $130.8,127.3,127.2,123.9,123.4,122.1,121.6,119.7,118.6,117.9,110.3,109.6,89.2,17.9$. ESI MS: $m / z 275$ 
$[\mathrm{M}+\mathrm{H}]^{+}$. Elemental analysis calcd (\%) for $\mathrm{C}_{18} \mathrm{H}_{14} \mathrm{~N}_{2} \mathrm{O}$ : C 78.81; $\mathrm{H}$ 5.14; $\mathrm{N} 10.21$; found: $\mathrm{C} 78.77 ; \mathrm{H}$ 5.16 ; N 10.30 .

2-(7-Methylpyrido[2,3-b]indolizin-2-yl)phenol (31). Prepared according to the Method C. Brown powder; m.p. 226-227 ${ }^{\circ} \mathrm{C}$ (decomp.); yield, $26 \mathrm{mg}(24 \%) ;{ }^{1} \mathrm{H}$ NMR (400 MHz, $\left.\mathrm{CDCl}_{3}\right) \delta 15.13$ (s, 1H, OH), 8.81 (s, $1 \mathrm{H}, \mathrm{H}-6), 8.78$ (d, J = 8.9 Hz, 1H, H-3), $8.11(\mathrm{~d}, J=7.1 \mathrm{~Hz}, 1 \mathrm{H}, \mathrm{Ph}-\mathrm{H}), 8.03$ (d, $J=8.9 \mathrm{~Hz}, 1 \mathrm{H}, \mathrm{H}-4), 7.60$ (d, J = 9.1 Hz, 1H, H-9), 7.28-7.30 (m, 1H, Ph-H), 7.07 (d, J = 9.1 Hz, 1H, H-8), 6.91-6.95 (m, 2H, Ph-H), 6.78 (s, 1H, H-10), 2.30 (s, 3H, C $\left.{ }_{9}-\mathrm{CH}_{3}\right) .{ }^{13} \mathrm{C} \mathrm{NMR}\left(100 \mathrm{MHz}, \mathrm{CDCl}_{3}\right) \delta$ 159.5, 154.1, 141.4, 138.3, 130.7, 128.4, 127.2, 123.2, 121.3, 121.2, 119.7, 118.6 (2C), 118.3, 117.8, 110.0, 89.8, 17.7. ESI MS: $m / z 275[\mathrm{M}+\mathrm{H}]^{+}$. Elemental analysis calcd (\%) for $\mathrm{C}_{18} \mathrm{H}_{14} \mathrm{~N}_{2} \mathrm{O}$ : C 78.81; H 5.14; $\mathrm{N} 10.21$; found: C 78.83; $\mathrm{H}$ 5.11; $\mathrm{N} 10.28$.

2-(Pyridin-4-yl)pyrido[2,3-b]indolizine (3m). Prepared according to the Method A. Eluent ethyl acetate-hexane 1: 5. Orange powder; m.p. $196-199{ }^{\circ} \mathrm{C}$ (decomp.); yield, $30 \mathrm{mg}(31 \%) ;{ }^{1} \mathrm{H} \mathrm{NMR}\left(400 \mathrm{MHz}, \mathrm{CDCl}_{3}\right) \delta 8.73$ $(\mathrm{d}, J=5.9 \mathrm{~Hz}, 2 \mathrm{H}, \mathrm{Py}-\mathrm{H}), 8.29(\mathrm{~d}, J=6.6 \mathrm{~Hz}, 1 \mathrm{H}, \mathrm{H}-6), 8.21(\mathrm{~d}, J=8.6 \mathrm{~Hz}, 1 \mathrm{H}, \mathrm{H}-3), 8.01(\mathrm{~d}, J=5.9 \mathrm{~Hz}, 2 \mathrm{H}$, Py-H), 7.66 (d, J = 8.6 Hz, 1H, H-4), 7.52 (d, J = 9.6 Hz, 1H, H-9), 7.00-7.03 (m, 1H, H-7), 6.90 (s, 1H, H-10), 6.58-6.60 (m, 1H, H-8). ${ }^{13} \mathrm{C}$ NMR (100 MHz, $\left.\mathrm{CDCl}_{3}\right) \delta 151.7,150.2$ (2C), 147.7, 146.5, 139.9, 124.7, 124.0, 122.4, $121.7(2 \mathrm{C}), 119.6,118.2,111.8,109.2,92.7$. ESI MS: $m / z 246[\mathrm{M}+\mathrm{H}]^{+}$. Elemental analysis calcd (\%) for $\mathrm{C}_{16} \mathrm{H}_{11} \mathrm{~N}_{3}$ : C 78.35; $\mathrm{H}$ 4.52; N 17.13; found: C 78.31; $\mathrm{H} 4.55 ; \mathrm{N} 17.20$.

9-Methyl-2-(pyridin-4-yl)pyrido[2,3-b]indolizine (3n). Prepared according to the Method A. Eluent ethyl acetate-hexane 1: 5 . Light beige powder; m.p. $173-174{ }^{\circ} \mathrm{C}$ (decomp.); yield, $34 \mathrm{mg}(33 \%) ;{ }^{1} \mathrm{H} \mathrm{NMR}$ $\left(400 \mathrm{MHz}, \mathrm{CDCl}_{3}\right) \delta 8.72(\mathrm{~d}, J=5.8 \mathrm{~Hz}, 1 \mathrm{H}, \mathrm{Py}-\mathrm{H}), 8.18-8.20(\mathrm{~m}, 2 \mathrm{H}, \mathrm{H}-3, \mathrm{H}-6), 8.01(\mathrm{~d}, J=5.8 \mathrm{~Hz}$, 2H, Py-H), $7.65(\mathrm{~d}, J=8.6 \mathrm{~Hz}, 1 \mathrm{H}, \mathrm{H}-4), 6.86(\mathrm{~s}, 1 \mathrm{H}, \mathrm{H}-10), 6.82(\mathrm{~d}, J=6.6 \mathrm{~Hz}, 1 \mathrm{H}, \mathrm{H}-8), 6.54-6.56(\mathrm{~m}$, 1H, H-7), 3.18 (s, 3H, $\left.\mathrm{C}_{9}-\mathrm{CH}_{3}\right) .{ }^{13} \mathrm{C}$ NMR (100 MHz, $\left.\mathrm{CDCl}_{3}\right) \delta 151.5,150.5,150.2(2 \mathrm{C}), 147.7,141.1$, 128.5, 123.6, 122.5, 122.3, $121.6(2 \mathrm{C}), 118.3,111.8,109.3,91.2,18.5$. ESI MS: $m / z 260[\mathrm{M}+\mathrm{H}]^{+}$. Elemental analysis calcd (\%) for $\mathrm{C}_{17} \mathrm{H}_{13} \mathrm{~N}_{3}$ : C 78.74; $\mathrm{H} 5.05 ; \mathrm{N} 16.20$; found: C 78.71; H 5.09; $\mathrm{N} 16.29$.

7-Methyl-2-(pyridin-4-yl)pyrido[2,3-b]indolizine (3o). Prepared according to the Method A. Eluent ethyl acetate-hexane 1: 5. Light beige powder; m.p. $223-225^{\circ} \mathrm{C}$ (decomp.); yield, $27 \mathrm{mg}(26 \%) ;{ }^{1} \mathrm{H} \mathrm{NMR}$ $\left(400 \mathrm{MHz}, \mathrm{CDCl}_{3}\right) \delta 8.73(\mathrm{~d}, J=5.8 \mathrm{~Hz}, 2 \mathrm{H}, \mathrm{Py}-\mathrm{H}), 8.21(\mathrm{~d}, J=8.6 \mathrm{~Hz}, 1 \mathrm{H}, \mathrm{H}-3), 8.11(\mathrm{~s}, 1 \mathrm{H}, \mathrm{H}-6), 8.03$ $(\mathrm{d}, J=5.8 \mathrm{~Hz}, 2 \mathrm{H}, \mathrm{Py}-\mathrm{H}), 7.66(\mathrm{~d}, J=8.6 \mathrm{~Hz}, 1 \mathrm{H}, \mathrm{H}-4), 7.47$ (d, J = 8.9 Hz, 1H, H-9), 6.90 (d, J = $8.9 \mathrm{~Hz}$, $1 \mathrm{H}, \mathrm{H}-8), 6.87$ (s, 1H, H-10), 2.33 (s, 3H, $\left.\mathrm{C}_{7}-\mathrm{CH}_{3}\right) .{ }^{13} \mathrm{C} \mathrm{NMR}\left(100 \mathrm{MHz}, \mathrm{CDCl}_{3}\right) \delta$ 151.3, 150.1 (2C), 147.9, 146.4, 139.0, 127.6, 122.2, 121.9, $121.7(2 \mathrm{C}), 119.1,118.6,118.1,111.6,92.2,18.3$. ESI MS: $m / z 260[\mathrm{M}+\mathrm{H}]^{+}$. Elemental analysis calcd (\%) for $\mathrm{C}_{17} \mathrm{H}_{13} \mathrm{~N}_{3}$ : C 78.74; $\mathrm{H}$ 5.05; $\mathrm{N} 16.20$; found: C 78.69; $\mathrm{H}$ 5.06; N 16.32.

2-(Pyridin-2-yl)pyrido[2,3-b]indolizine (3p). Prepared according to the Method A. Eluent ethyl acetate-hexane 1: 10. Yellow powder; m.p. $159-162{ }^{\circ} \mathrm{C}$ (decomp.); yield, $27 \mathrm{mg}(28 \%) ;{ }^{1} \mathrm{H} \mathrm{NMR}$ $\left(400 \mathrm{MHz}, \mathrm{CDCl}_{3}\right) \delta 8.72(\mathrm{~d}, J=4.0 \mathrm{~Hz}, 1 \mathrm{H}, \mathrm{Pyr}-\mathrm{H}), 8.60(\mathrm{~d}, J=8.1 \mathrm{~Hz}, 1 \mathrm{H}, \mathrm{Py}-\mathrm{H}), 8.38(\mathrm{~d}, J=8.6 \mathrm{~Hz}, 1 \mathrm{H}$, H-3), 8.33 (d, J = 7.1 Hz, 1H, H-6), 8.28 (d, J = 8.6 Hz, 1H, H-4), $7.86(\mathrm{~m}, 1 \mathrm{H}, \mathrm{Py}-\mathrm{H}), 7.52(\mathrm{~d}, J=9.1 \mathrm{~Hz}$, 1H, H-9), 7.31-7.33 (m, 1H, Py-H), 6.99-7.01 (m, 1H, H-7), 6.91 (s, 1H, H-10), 6.58-6.60 (1H, м, H-8), 6.58-6.60 (m, 1H, H-8). ${ }^{13} \mathrm{C}$ NMR (100 MHz, $\left.\mathrm{CDCl}_{3}\right) \delta 157.2,153.4,149.0,145.9,139.5,136.9,124.7$, 123.6, 123.3, 122.8, 121.5, 119.5, 118.2, 112.5, 108.9, 92.5. ESI MS: $m / z 246[\mathrm{M}+\mathrm{H}]^{+}$. Elemental analysis calcd (\%) for $\mathrm{C}_{16} \mathrm{H}_{11} \mathrm{~N}_{3}$ : C 78.35; $\mathrm{H} 4.52 ; \mathrm{N} 17.13$; found: $\mathrm{C} 78.32 ; \mathrm{H} 4.57 ; \mathrm{N}$ 17.14.

9-Methyl-2-(pyridin-2-yl)pyrido[2,3-b]indolizine (3q). Prepared according to the Method A. Eluent ethyl acetate-hexane 1: 10. Lime-green powder; m.p. $124-127^{\circ} \mathrm{C}$ (decomp.); yield, $71 \mathrm{mg}(70 \%) ;{ }^{1} \mathrm{H} \mathrm{NMR}$ $\left(400 \mathrm{MHz}, \mathrm{CDCl}_{3}\right) \delta 8.71(\mathrm{~d}, J=4.0 \mathrm{~Hz}, 1 \mathrm{H}, \mathrm{Py}-\mathrm{H}), 8.61(\mathrm{~d}, J=7.6 \mathrm{~Hz}, 1 \mathrm{H}, \mathrm{Py}-\mathrm{H}), 8.37(\mathrm{~d}, J=8.6 \mathrm{~Hz}$, 1H, H-3), 8.25 (d, J = 8.6 Hz, 1H, H-4), 8.22 (d, J = 7.1 Hz, 1H, H-6), 7.84-7.87 (m, 1H, Py-H), 7.30-7.32 (m, 1H, Py-H), 6.88 (s, 1H, H-10), 6.80 (d, J = 6.1 Hz, 1H, H-8), 6.53-6.55 (m, 1H, H-7), $2.51(\mathrm{~s}, 3 \mathrm{H}$, $\left.\mathrm{C}_{9}-\mathrm{CH}_{3}\right) .{ }^{13} \mathrm{C}$ NMR $\left(100 \mathrm{MHz}, \mathrm{CDCl}_{3}\right) \delta 157.2,153.3,149.0,145.9,140.7,136.8,128.4,123.4,123.2,122.4$, 122.1, 121.5, 118.3, 112.5, 109.1, 91.1, 18.5. ESI MS: $m / z 260[\mathrm{M}+\mathrm{H}]^{+}$. Elemental analysis calcd (\%) for $\mathrm{C}_{17} \mathrm{H}_{13} \mathrm{~N}_{3}$ : C 78.74; H 5.05; N 16.20; found: C 78.70; H 5.07; N 16.25. 
7-Methyl-2-(pyridin-4-yl)pyrido[2,3-b]indolizine (3r). Prepared according to the Method A. Eluent ethyl acetate-hexane 1: 7. Brown powder; m.p. $74-79{ }^{\circ} \mathrm{C}$ (decomp.); yield, $39 \mathrm{mg}(38 \%) ;{ }^{1} \mathrm{H} \mathrm{NMR}(400 \mathrm{MHz}$, $\left.\mathrm{CDCl}_{3}\right) \delta 8.71(\mathrm{~d}, J=4.0 \mathrm{~Hz}, 1 \mathrm{H}, \mathrm{Py}-\mathrm{H}), 8.59(\mathrm{~d}, J=8.1 \mathrm{~Hz}, 1 \mathrm{H}, \mathrm{Py}-\mathrm{H}), 8.35(\mathrm{~d}, J=8.6 \mathrm{~Hz}, 1 \mathrm{H}, \mathrm{H}-3), 8.25$ $(\mathrm{d}, J=8.6 \mathrm{~Hz}, 1 \mathrm{H}, \mathrm{H}-4), 8.13$ (s, 1H, H-6), 7.83-7.86 (m, 1H, Py-H), 7.47 (d, J = 9.1 Hz, 1H, H-9), 7.30-7.32 (m, 1H, Py-H), 6.86-6.89 (m, 2H, H-8, H-10), 2.33 (s, 3H, C $\left.7-\mathrm{CH}_{3}\right) .{ }^{13} \mathrm{C} \mathrm{NMR}\left(100 \mathrm{MHz}, \mathrm{CDCl}_{3}\right) \delta 157.4$, 153.2, 149.0, 146.0, 138.5, 136.8, 127.1, 123.2, 122.6, 121.9, 121.5, 119.0, 118.2, 118.0, 112.3, 92.1, 18.3. ESI MS: $m / z 260[\mathrm{M}+\mathrm{H}]^{+}$. Elemental analysis calcd (\%) for $\mathrm{C}_{17} \mathrm{H}_{13} \mathrm{~N}_{3}$ : C 78.74; $\mathrm{H}$ 5.05; N 16.20; found: $\mathrm{C}$ 78.71; H 5.09; N 16.33 .

\section{Conclusions}

In conclusion, we discovered a novel domino route to condensed indolizines-pyrido[2,3-b] indolizines, containing various aromatic or heteroaromatic moieties at $C(2)$ and alkyl groups at $C(7)$ or $\mathrm{C}(9)$. The route is based on the interaction of 2-alkyl-N-(cyanomethyl)pyridinium salts with enaminones. The synthesized compounds are effective fluorophores, emitting green light with FQYs up to $82 \%$.

Supplementary Materials: The supplementary materials are available online.

Author Contributions: Conceptualization, L.G.V. and E.V.V.d.E.; methodology, E.A.S., A.V.V. and A.A.F.; X-ray analysis, V.B.R.; optical studies, K.S.; writing—original draft preparation, A.A.F.; writing-review and editing, L.G.V. and E.V.V.d.E. All authors have read and agreed to the published version of the manuscript.

Funding: The publication was prepared with the support of the "RUDN University Program 5-100" and RFBR Grant 18-33-20101 (Sokolova E.A.). Funding for optical studies was provided by the Ministry of Science and Higher Education of the Russian Federation (award no. 075-03-2020-223 (FSSF-2020-0017)).

Acknowledgments: The research is carried out using the equipment of the shared research facilities of HPC computing resources at Lomonosov Moscow State University.

Conflicts of Interest: The authors declare no conflict of interest.

\section{References}

1. Kappert, F.; Sreeramulu, S.; Jonker, H.R.A.; Richter, C.; Rogov, V.V.; Proschak, E.; Hargittay, B.; Saxena, K.; Schwalbe, H. Structural characterization of the interaction of the fibroblast growth factor receptor with a small molecule allosteric inhibitor. Chemistry 2018, 24, 7861-7865. [CrossRef] [PubMed]

2. Yu, Q.; Yang, H.; Zhu, T.; Yu, L.; Chen, J.; Gu, L.; Huang, Z.; An, L. Synthesis, cytotoxicity and structure-activity relationship of indolizinoquinolinedione derivatives as DNA topoisomerase IB catalytic inhibitors. Eur. J. Med. Chem. 2018, 152, 195-207. [CrossRef] [PubMed]

3. Park, S.; Kim, E.H.; Kim, J.; Kim, S.H.; Kim, I. Biological evaluation of indolizine-chalcone hybrids as new anticancer agents. Eur. J. Med. Chem. 2018, 144, 435-443. [CrossRef]

4. Tatipamula, V.B.; Kolli, M.K.; Lagu, S.B.; Paidi, K.R.; Rsddy, R.; Yejella, R.P. Novel indolizine derivatives lowers blood glucose levels in streptozotocin-induced diabetic rats: A histopathological approach. Pharm. Rep. 2019, 71, 233-242. [CrossRef] [PubMed]

5. Moon, S.H.; Jung, Y.; Kim, S.H.; Kim, I. Synthesis, characterization and biological evaluation of anti-cancer indolizine derivatives via inhibiting $\beta$-catenin activity and activating p53. Bioorg. Med. Chem. Lett. 2016, 26, 110-113. [CrossRef]

6. Sardaru, M.C.; Craciun, A.M.; Al Matarneh, C.M.; Sandu, I.A.; Amarandi, R.M.; Popovici, L.; Ciobanu, C.I.; Peptanariu, D.; Pinteala, M.; Mangalagiu, I.I.; et al. Cytotoxic substituted indolizines as new colchicine site tubulin polymerisation inhibitors. J. Enzym. Inhib. Med. Chem. 2020, 35, 1581-1595. [CrossRef]

7. Albaladejo, M.J.; González-Soria, M.J.; Alonso, F. Metal-free remote-site C-H alkenylation: Regio- and diastereoselective synthesis of solvatochromic dyes. Green Chem. 2018, 20, 701-712. [CrossRef]

8. Gayton, J.; Autry, S.A.; Meador, W.; Parkin, S.R.; Hill, G.A.; Hammer, N.I.; Delcamp, J.H. Indolizine-Cyanine dyes: Near infrared emissive cyanine dyes with increased Stokes shifts. J. Org. Chem. 2019, 84, 687-697. [CrossRef] 
9. Cheema, H.; Baumann, A.; Loya, E.K.; Brogdon, P.; McNamara, L.E.; Carpenter, C.A.; Hammer, N.I.; Mathew, S.; Risko, C.; Delcamp, J.H. Near-Infrared-Absorbing indolizine-porphyrin push-pull dye for dye-sensitized solar cells. ACS Appl. Mater. Interfaces 2019, 11, 16474-16489. [CrossRef]

10. Yang, J.; Zhu, Y.; Tse, A.K.W.; Zhou, X.; Chen, Y.; Tse, Y.C.; Wong, K.M.C.; Ho, C.Y. Synthesis and study of $\mathrm{Au}(\mathrm{iii})$-indolizine derivatives: Turn-on luminescence by photo-induced controlled release. Chem. Commun. 2019, 55, 4471-4474. [CrossRef]

11. Lee, Y.; Cho, W.; Sung, J.; Kim, E.; Park, S.B. Monochromophoric design strategy for tetrazine-based colorful bioorthogonal probes with a single fluorescent core skeleton. J. Am. Chem. Soc. 2018, 140, 974-983. [CrossRef] [PubMed]

12. Ji, R.; Liu, A.; Shen, S.; Cao, X.; Li, F.; Ge, Y. An indolizine-rhodamine based FRET fluorescence sensor for highly sensitive and selective detection of $\mathrm{Hg}^{2+}$ in living cells. RSC Adv. 2017, 7, 40829-40833. [CrossRef]

13. Bayazit, M.K.; Pålsson, L.O.; Coleman, K.S. Sensing properties of light-emitting single walled carbon nanotubes prepared via click chemistry of ylides bound to the nanotube surface. RSC Adv. 2015, 5, 36865-36873. [CrossRef]

14. Airinei, A.; Tigoianu, R.; Danac, R.; Al Matarneh, C.M.; Isac, D.L. Steady state and time resolved fluorescence studies of new indolizine derivatives with phenanthroline skeleton. J. Lumin. 2018, 199, 6-12. [CrossRef]

15. Kucukdisli, M.; Opatz, T. A modular synthesis of polysubstituted indolizines. Eur. J. Org. Chem. 2012, 2012, 4555-4564. [CrossRef]

16. Cai, Q.; Zhu, Y.P.; Gao, Y.; Sun, J.J.; Wu, A.X. A direct method for the synthesis of indolizine derivatives from easily available aromatic ketones, pyridines, and acrylonitrile derivatives. Can. J. Chem. 2013, 91, 414-419. [CrossRef]

17. Wang, W.; Han, J.; Sun, J.; Liu, Y. CuBr-Catalyzed aerobic decarboxylative cycloaddition for the synthesis of indolizines under solvent-free conditions. J. Org. Chem. 2017, 82, 2835-2842. [CrossRef]

18. Wang, D.; Zhang, X.; He, C.; Duan, C. Aminonaphthalimide-based imidazolium podands for turn-on fluo. Org. Biomol. Chem. 2010, 8, 2873-3084. [CrossRef]

19. Bonte, S.; Ghinea, I.O.; Dinica, R.; Baussanne, I.; Demeunynck, M. Investigation of the pyridinium ylide-alkyne cycloaddition as a fluorogenic coupling reaction. Molecules 2016, 21, 332. [CrossRef]

20. Yavari, I.; Ghafouri, K.; Naeimabadi, M.; Halvagar, M.R. A synthesis of functionalized 2-Indolizin-3-yl-1,3benzothiazoles from 1-(1,3-Benzothiazol-2-ylmethyl)pyridinium Iodide and Acetylenic Esters. Synlett 2018, 29, 243-245. [CrossRef]

21. Yavari, I.; Sheykhahmadi, J.; Naeimabadi, M.; Halvagar, M.R. Iodine-mediated $\mathrm{sp}^{3}$ C-H functionalization of methyl ketones: A one-pot synthesis of functionalized indolizines via the 1,3-dipolar cycloaddition reaction between pyridinium ylides and ynones. Mol. Divers. 2017, 21, 1-8. [CrossRef] [PubMed]

22. Douglas, T.; Pordea, A.; Dowden, J. Iron-Catalyzed indolizine synthesis from pyridines, diazo compounds, and alkynes. Org. Lett. 2017, 19, 6396-6399. [CrossRef] [PubMed]

23. Shang, Y.; Zhang, M.; Yu, S.; Ju, K.; Wang, C.; He, X. New route synthesis of indolizines via 1,3-dipolar cycloaddition of pyridiniums and alkynes. Tetrahedron Lett. 2009, 50, 6981-6984. [CrossRef]

24. Fu, Q.; Yan, C.G. Molecular diversity of cycloaddition reactions of the functionalized pyridinium salts with 3-phenacylideneoxindoles. Tetrahedron 2013, 69, 5841-5849. [CrossRef]

25. Zheng, P.; Li, C.; Mou, C.; Pan, D.; Wu, S.; Xue, W.; Jin, Z.; Chi, Y.R. Efficient access to 2-Pyrones via carbene-catalyzed oxidative [3+3] reactions between enals and nitrogen Ylides. Asian J. Org. Chem. 2019, 8, 1067-1070. [CrossRef]

26. Zhang, Y.F.; Duan, W.D.; Chen, J.; Hu, Y. Base-Promoted cascade reactions of 3-(1-Alkynyl)chromones with pyridinium Ylides to Chromeno[2,3-D]azepine derivatives. J. Org. Chem. 2019, 84, 4467-4472. [CrossRef]

27. Kucukdisli, M.; Opatz, T. Two-step synthesis of 2-aminoindolizines from 2-alkylpyridines. Eur. J. Org. Chem. 2014, 2014, 5836-5844. [CrossRef]

28. Storozhenko, O.A.; Festa, A.A.; Ndoutoume, D.R.B.; Aksenov, A.V.; Varlamov, A.V.; Voskressensky, L.G. Mn-mediated sequential three-component domino Knoevenagel/cyclization/Michael addition/oxidative cyclization reaction towards annulated imidazo[1,2-a]pyridines. Beilstein J. Org. Chem. 2018, 14, 3078-3087. [CrossRef]

29. Voskressensky, L.G.; Storozhenko, O.A.; Festa, A.A.; Novikov, R.A.; Varlamov, A.V. Synthesis of Chromenoimidazoles, annulated with an azaindole moiety, through a base-promoted domino reaction of cyano methyl quaternary salts. Synthesis 2017, 49, 2753-2760. 
30. Voskressensky, L.G.; Sokolova, E.A.; Festa, A.A.; Varlamov, A.V. A novel domino condensation-intramolecular nucleophilic cyclization approach towards annulated thiochromenes. Tetrahedron Lett. 2013, 54, 5172-5173. [CrossRef]

31. Sokolova, E.A.; Festa, A.A.; Golantsov, N.E.; Lukonina, N.S.; Ioffe, I.N.; Varlamov, A.V.; Voskressensky, L.G. Highly fluorescent pyrido[2,3-b]indolizine-10-carbonitriles through pseudo three-component reactions of N-(Cyanomethyl)pyridinium salts. Eur. J. Org. Chem. 2019, 2019, 6770-6775. [CrossRef]

32. Kro, R.; Mostafavi, M.; Lampre, I. Preferential solvation of coumarin 153. The role of hydrogen bonding. J. Phys. Chem. A 2002, 106, 1708-1713.

33. Shen, Y.M.; Grampp, G.; Leesakul, N.; Hu, H.W.; Xu, J.H. Synthesis and emitting properties of the blue-light fluorophores indolizino[3,4,5-ab]isoindole derivatives. Eur. J. Org. Chem. 2007, 2007, 3718-3726. [CrossRef]

34. Park, S.; Kwon, D.I.; Lee, J.; Kim, I. When indolizine meets quinoline: Diversity-oriented synthesis of new polyheterocycles and their optical properties. ACS Comb. Sci. 2015, 17, 459-469. [CrossRef]

35. Singh, D.K.; Kim, S.; Lee, J.H.; Lee, N.K.; Kim, J.; Lee, J.; Kim, I. 6-(Hetero)arylindolizino[1,2-c]quinolines as highly fluorescent chemical space: Synthesis and photophysical properties. J. Heterocycl. Chem. 2020, 57, 3018-3028. [CrossRef]

36. Sung, J.; Lee, Y.; Cha, J.H.; Park, S.B.; Kim, E. Development of fluorescent mitochondria probe based on 1,2-dihydropyrrolo[3,4-b]indolizine-3-one. Dyes Pigments 2017, 145, 461-468. [CrossRef]

37. Al-Zaydi, K.M.; Borik, R.M. Microwave assisted condensation reactions of 2-aryl hydrazonopropanals with nucleophilic reagents and dimethyl acetylenedicarboxylate. Molecules 2007, 12, 2061-2079. [CrossRef]

Sample Availability: Not available.

(C) 2020 by the authors. Licensee MDPI, Basel, Switzerland. This article is an open access article distributed under the terms and conditions of the Creative Commons Attribution (CC BY) license (http://creativecommons.org/licenses/by/4.0/). 